\title{
SOME ASPECTS OF SOLID STATE RADIOLUMINESCENCE
}

\author{
A.J. WoJTOWICZ \\ Institute of Physics, N. Copernicus University, Grudziądzka 5, 87-100 Toruń, Poland \\ and \\ Boston University, Chemistry Department \\ 590 Commonwealth Ave., Boston, MA 02215, USA
}

\begin{abstract}
In this paper we review results of radioluminescence studies on two scintillator materials, $\mathrm{LuAlO}_{3}$ and $\mathrm{YAlO}_{3}$, activated with Ce. The experiments include measurements of thermoluminescence, isothermal phosphorescence decays, scintillation light yield as function of temperature, and scintillation time profiles under gamma excitation. Experimental results are interpreted in the frame of a simple kinetic model that includes a number of electron traps. We have identified and characterized a number of deep and shallow traps and demonstrated that traps in $\mathrm{LuAlO}_{3}: \mathrm{Ce}$ are deeper than corresponding traps in $\mathrm{YAlO}_{3}: \mathrm{Ce}$. Unlike deep traps which are responsible for some scintillation light loss but otherwise do not have any impact on generation of scintillation light, shallow traps are shown to actively interfere with the process of radiative recombination via $\mathrm{Ce}$ ions. We demonstrate that shallow traps are responsible for some as yet unexplained observations including a higher room temperature light yield of $\mathrm{YAlO}_{3}: \mathrm{Ce}$ and its longer scintillation decay time, as well as a longer scintillation rise time in $\mathrm{LuAlO}_{3}: \mathrm{Ce}$.
\end{abstract}

PACS numbers: 78.60.Ya, 78.30.Hv, 78.60.Kn, 29.40.Mc

\section{Introduction}

The term radioluminescence was coined to describe light emission excited by radioactive substances or, more precisely, by ionizing radiation $\left(\alpha^{-}, \beta-\right.$, and $\gamma$-particles) emanating from them [1]. Indeed, it was the radioluminescence of uranyl salts that facilitated the discovery of radioactivity itself by Becquerel in 1896. Tiny visible "scintillations" produced by single $\alpha$-particles in radioluminescent material (ZnS:Ag), observed by Rutherford in his famous experiment, not only provided a detection method for high energy physics but also gave a name to a class of radioluminescent materials: scintillators. Another application of radioluminescence, in fluorescent screens to detect X-rays, leads to a different albeit closely related class of materials, X-ray phosphors, without which medical radiology would be far more difficult and dangerous. 
Scintillator materials are widely used not only in detectors for high energy and nuclear physics, but in industry (quality control, oil exploration, airport security) and medicine ( $\gamma$-ray detectors for positron emission tomography (PET), computer tomography (CT)) as well. A number of materials have a long history of use in such applications; these include $\mathrm{ZnS}: \mathrm{Ag}, \mathrm{NaI}: \mathrm{Tl}, \mathrm{CsI}: \mathrm{Tl}, \mathrm{CaF}_{2}: \mathrm{Eu}$, which are characterized by relatively low density of $3.2-4.5 \mathrm{~g} / \mathrm{cm}^{3}$, slow decays of scintillation pulse (hundreds of ns to a few $\mu \mathrm{s}$ ) and reasonably high light yield of up to 52000 visible photons per $\mathrm{MeV}$ of energy deposited by a high-energy particle such as a $\gamma$-photon. An important addition to this group was BGO $\left(\mathrm{Bi}_{4} \mathrm{Ge}_{3} \mathrm{O}_{12}\right)$, whose high density $\left(7.13 \mathrm{~g} / \mathrm{cm}^{3}\right)$ enabled efficient stopping of $0.5 \mathrm{MeV} \gamma$-particles used in PET. However, its relatively low light yield ( 8000 photons $/ \mathrm{MeV}$ ) and speed (about $360 \mathrm{~ns}$ ) leave much to be desired.

With all of the established materials falling short of the stringent timing requirements in modern applications, a worldwide search for new scintillator materials was initiated in the late eighties. These efforts led to the discovery of a number of promising new materials, characterized by reasonably high density, high light yield, and fast decay and rise times. The most prominent of these, and the most developed, is $\mathrm{LSO}: \mathrm{Ce}\left(\mathrm{Lu}_{2} \mathrm{SiO}_{5}: \mathrm{Ce}\right)$, discovered by Melcher and Schweitzer in 1992 [2]. Another material with attractive properties, but far less advanced in development, is $\mathrm{LuAP}: \mathrm{Ce}\left(\mathrm{LuAlO}_{3}: \mathrm{Ce}\right)$, of which Czochralski-grown monocrystals were first evaluated in the garnet-free perovskite phase by Lempicki and collaborators in 1994 [3]. In addition, YAP:Ce $\left(\mathrm{YAlO}_{3}: \mathrm{Ce}\right)$, less dense but much cheaper than its Lu-analog, also attracts considerable interest (see e.g. [4]).

Wide band gap materials have long been the subject of active research as scintillator detectors of ionizing radiation. Such materials, when activated with some open $f$-shell rare earth ions, display Stokes-shifted VUV and UV $d-f$ emissions characterized by fast radiative decay rates and high quantum efficiencies. In X- or $\gamma$-ray detector materials, the role of the host is threefold: it must stop and convert an incoming high energy particle into a large number of electron-hole pairs; it must support an efficient mechanism by which this energy can migrate to the emitting center; and it must provide a favorable environment for the activator ion to luminesce efficiently. Of these three conditions, it is the efficient energy transfer that is the most demanding [5].

The primary mechanism by which the energy of electron-hole pairs is transferred to the activator ions in wide band gap materials is not fully understood. It is well-known that the effective charge states of open $d$ - or $f$-shell ions in insulating materials can readily be changed through interaction with charge carriers of either sign. Thus, despite the insulating nature of the host material, such ions may perform as efficient recombination centers for these carriers [6], suggesting that the consecutive trapping of charge carriers, a well-known mechanism in photoconductive materials such as $\mathrm{ZnS}: \mathrm{Ag}$ [7], should be an efficient energy transfer mechanism in activated wide band gap insulator materials. Indeed, despite earlier reservations [1], such a mechanism was proposed and applied successfully some time ago for Tl-activated scintillators (see e.g. Ref. [8]) and, more recently, for some Ce-based scintillators [9]. 
Despite the prominence of carrier recombination, however, other mechanisms of energy transfer may also play a role. Specifically, a high rate of exciton generation could conceivably promote a mechanism involving free exciton diffusion, as proposed in the early literature (see e.g. [1]). Also, the high polaron mass commonly found in wide band gap and strongly ionic materials should lead to efficient trapping and self-trapping of free charge carriers as well as excitons. Since excitonic effects such as defect- and self-trapped excitonic emissions [10] often dominate in undoped materials, their enhancement in cerium-doped oxide materials could lead to nonradiative energy transfer from the trapped excitons to the Ce ions, depending on the overlap between those defect emissions and the Ce-absorptions [11]. While this could not be a primary mechanism (see e.g. Ref. [12]), it is nevertheless true that higher concentrations of activating ions may enhance some secondary transfer processes.

In this paper we present a study of radioluminescence mechanism in two important scintillator oxide materials, LuAP:Ce and YAP:Ce, both of which have a band gap of more than $8 \mathrm{eV}$. We review recent measurements of thermoluminescence below and above $300 \mathrm{~K}$ (denoted low-temperature (ltTL) and high-temperature (htTL), respectively), scintillation light yield as function of temperature ( $L Y$ vs. $T$ ), and scintillation time profiles, all of which were performed at collaborating laboratories in Boston (Chemistry Department, Boston University) and Toruń (Institute of Physics, N. Copernicus University), as well as fast scintillation rise times measured at Lawrence Berkeley Laboratory in California [13]. For the first time all these experiments have been consistently interpreted in the frame of a simple model involving luminescence centers and a number of electron traps [14-16]. While the mobility of charge carriers may well be quite low, the success of this model provides a strong argument for a scintillation mechanism involving some kind of charge transport.

\section{Basic properties of LuAP:Ce and YAP:Ce scintillators}

Despite very different atomic masses $\mathrm{Y}^{3+}$ and $\mathrm{Lu}^{3+}$ ions have quite similar ionic radii $(0.89$ and $0.85 \AA)$ and chemical properties. Consequently, it is reasonable to expect that LuAP:Ce and YAP:Ce would assume the same (perovskite) crystal structure and, upon doping with $\mathrm{Ce}^{3+}$, would show similar optical properties. The only expected difference would be the highly desirable and substantial increase in differential energy losses for high energy particles (stopping power) afforded by LuAP:Ce. This would clearly benefit all the applications using higher energy gammas such as, among others, in PET cameras.

As expected, all the spectra of LuAP:Ce and YAP:Ce are indeed almost exactly identical, with their room temperature (RT) emission dominated by a poorly resolved double emission band peaking at about $360 \mathrm{~nm}$. A number of structured broad bands are also present at lower wavelengths, in both absorption and luminescence excitation $[3,9]$. The decay of the Ce-emission of both materials, under optical excitation directly into Ce-absorption bands, is almost exactly exponential, and the decay times are nearly identical, at about $16.8 \mathrm{~ns}$ (see citations in Ref. [16]). It was therefore an unexpected surprise to find that the scintillation properties of the two materials differ significantly. 
One major difference is in their scintillation time profiles. Despite their virtually indistinguishable optically excited profiles, LuAP:Ce and YAP:Ce exhibit quite disparate scintillation behavior. In both materials the scintillation decays are no longer single-exponential, but while the dominant component in LuAP:Ce has a characteristic time of about $17 \mathrm{~ns}$, essentially the same as the radiative decay time of the Ce ion, the corresponding component in the YAP:Ce scintillation decay is much slower, at least $25 \mathrm{~ns}$, and varies with Ce-concentration. Paradoxically, it is LuAP:Ce that has the slower rise time (0.6 ns, vs. $0.35 \mathrm{~ns}$ for YAP:Ce), as revealed by experiments of Derenzo and coworkers [13].

The two materials also differ in another important parameter, their light yield at RT, with the YAP:Ce value being some $50 \%$ higher $(17,100$ photons/MeV, vs. 11,300 for LuAP:Ce) [17]. Bartram et al. [18] suggested that the deep traps responsible for the htTL glow peaks between 300 and $600 \mathrm{~K}$ also contribute to the loss of scintillation light at RT. In their experiment they measured the ratio of thermoluminescence released during the heating cycle to the integrated steady state radioluminescence emitted during the preceding steady state $\gamma$-irradiation, finding values of 0.14 for LuAP:Ce and 0.02 for YAP:Ce. This implies that the YAP:Ce has considerably fewer deep traps into which carriers can be diverted. However, correction for such loss would raise the light yield of LuAP:Ce to $12,900 \mathrm{ph} / \mathrm{MeV}$, against 17,400 for YAP:Ce, still a substantial disparity. Clearly the deep traps are not by themselves adequate to explain the difference in the scintillation light yields at RT [15].

\section{High temperature thermoluminescence}

To explore the difference between the TL outputs of LuAP:Ce and YAP:Ce found by Bartram et al. [18] we have undertaken a detailed htTL study of both materials. This study included regular TL runs starting at $300 \mathrm{~K}$ (shown in Fig. 1), then runs that had been preceded by thermal annealing at increasingly higher temperatures and, in addition, a measurement of phosphorescence isothermal decays (ITD) [19].

Since a detailed report on these experiments and their interpretation has been given elsewhere [15] we will provide here only a brief summary. TL glow curves have been fitted to a set of four distinct traps using a TAMTAM procedure written by Bos et al. [20]. The procedure employs the expression

$$
I(T)=\sum_{i=1}^{4} n_{0 i} s_{i} \exp \left(-\frac{E_{i}}{k_{\mathrm{B}} T}\right) \exp \left[-\frac{s_{i}}{\beta} \int_{T_{0}}^{T} \exp \left(-\frac{E_{i}}{k_{\mathrm{B}} T}\right) \mathrm{d} T\right],
$$

where $\beta$ represents the heating rate, $k_{\mathrm{B}}$ the Boltzmann constant, $E_{i}$ the energy depths of the traps, $s_{i}$ their frequency factors, and $n_{0 i}$ the concentrations of occupied traps. This expression is derived from the well-known theory of Randall and Wilkins [21] extended to include four traps under the approximation of first-order kinetics [15]. Parameters retrieved by this procedure are summarized in Table I (LuAP:Ce) and Table II (YAP:Ce).

Thermal annealing and subsequent TL runs did not uncover any hidden or unresolved peaks; the ITD technique, however, did reveal a smaller TL glow peak in LuAP:Ce, hidden under the dominant peak at about $500 \mathrm{~K}$. In Fig. 2 we 


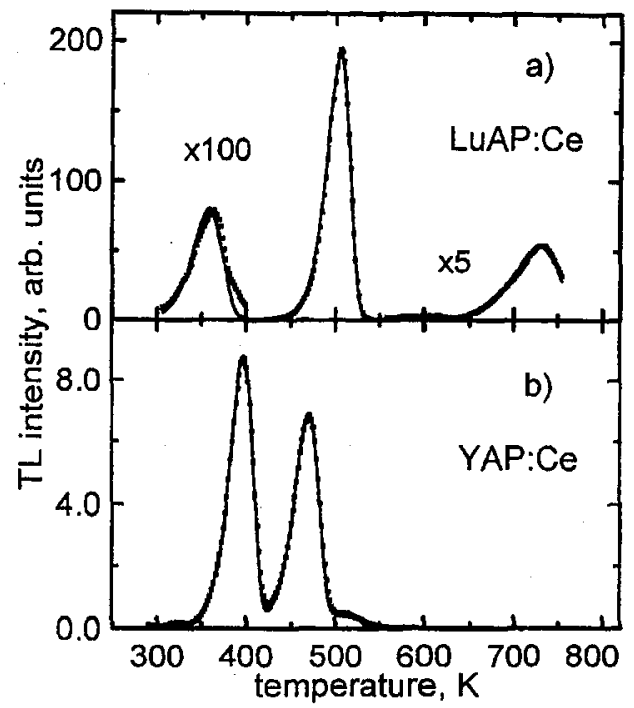

Fig. 1. Thermoluminescence glow curves of LuAP:Ce (a) (following $\beta$-irradiation for $2 \mathrm{~s}$ at RT) and YAP:Ce (b) (following $\beta$-irradiation for $5 \mathrm{~s}$ at RT). Squares represent experimental points, solid lines represent numerical fits (see text). The heating rate was $1 \mathrm{~K} \mathrm{~s}^{-1}$. Note the difference in intensity scales despite roughly comparable specimen size.

TABLE I

Summary of trap parameters in LuAP:Ce, derived from the glow curve fitting by the TAMTAM procedure for four deep traps.

\begin{tabular}{c|c|c|c}
\hline \hline Peak number $i$ & $n_{0 i} / n_{02}$ & $E_{i}[\mathrm{eV}]$ & $\ln s_{i}$ \\
\hline 1 & 0.005 & 0.65 & 18.2 \\
2 & 1.000 & 1.69 & 36.3 \\
3 & 0.006 & 1.23 & 21.1 \\
4 & 0.124 & 1.70 & 23.7
\end{tabular}

TABLE II

Summary of trap parameters in YAP:Ce, derived from the glow curve fitting by the TAMTAM procedure for four deep traps.

\begin{tabular}{c|c|c|c}
\hline \hline Peak number $i$ & $n_{0 i} / n_{02}$ & $E_{i}[\mathrm{eV}]$ & $\ln s_{i}$ \\
\hline 1 & 0.023 & 0.74 & 24.1 \\
2 & 1.000 & 1.04 & 27.7 \\
3 & 0.865 & 1.36 & 30.8 \\
4 & 0.073 & 1.42 & 29.3
\end{tabular}


plot phosphorescence decay times against the inverse temperature in the range covered by this peak. The mere presence of two components in ITD decays at those particular temperatures directly proves the existence of two different traps, with the parameters obtained from straight line fits to the experimental points in Fig. 2 fixing their frequency factors and energy depths [15].

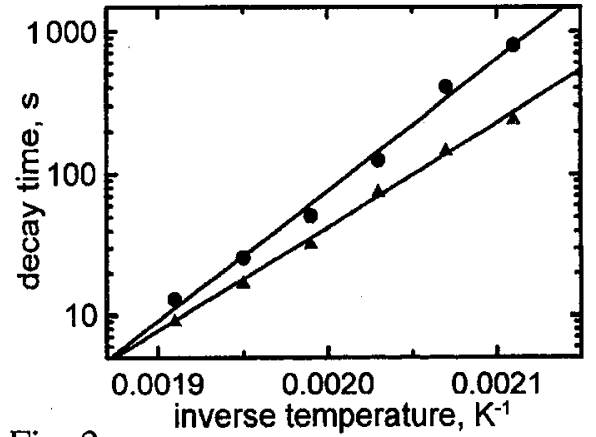

Fig. 2

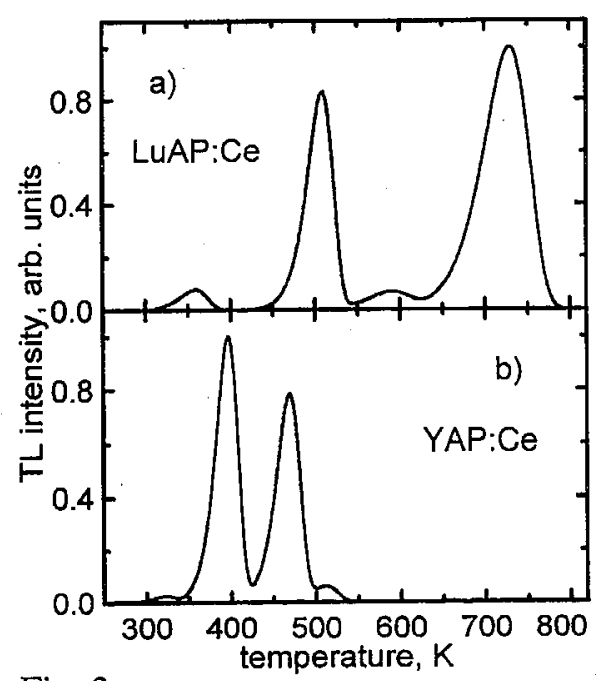

Fig. 3

Fig. 2. Semilog plots of LuAP:Ce phosphorescence isothermal decay times against inverse temperature. Decay times, shown by circles and triangles, were obtained from two-exponential fits to decay curves measured at various elevated temperatures in the range covered by the dominant TL peak, following $\beta$-irradiation at RT. The solid straight lines represent fits to experimental points, yielding trap depths and frequency factors.

Fig. 3. Simulated thermoluminescence glow curves of LuAP:Ce (a) and YAP:Ce (b) with the dominant glow peak of LuAP:Ce removed. The heating rate was $1 \mathrm{~K} \mathrm{~s}^{-1}$.

Since the TL of LuAP:Ce is so dominated by the $500 \mathrm{~K}$ peak (note the scales in Fig. 1), it would be desirable to remove its influence to get a clearer picture of the other features. This we do by calculating a simulated glow curve from formula (1) using parameters from TAMTAM and ITD fits. As seen in Fig. 3, the resulting TL glow curves of LuAP:Ce resemble those from YAP:Ce, except for a distinct shift to higher temperatures. With the frequency factors of all these traps falling relatively close to each other (in the range of $10^{13} \mathrm{~s}^{-1}$, as discussed in detail in Ref. [15]), the difference in positions of the glow peaks in the two materials must be largely attributed to the difference in their energy depths, implying that the traps in LuAP:Ce are deeper than their YAP:Ce counterparts.

As for the trap from which the dominant $500 \mathrm{~K}$ glow peak in LuAP:Ce originates, it is clear that it is largely responsible for the significantly greater TL output of this material, since there is no related counterpart trap in YAP:Ce. We also note that it has an unusually high frequency factor of about $10^{16} \mathrm{~s}^{-1}$, 
suggesting that the defect generating this trap is quite likely of a different nature. What this nature actually is, however, remains unknown.

\section{Scintillation light yield}

It has long been known that the photoluminescence yield at any given temperature is determined by the competition between radiative and nonradiative transitions from the luminescing level. Since thermally activated nonradiative processes are characterized by rates that increase exponentially with temperature, the temperature dependence of the photoluminescence light yield generally assumes the well known inverse sigmoid shape illustrated in Fig. $4 \mathrm{a}$ for $\mathrm{Ce}^{3+}$ in YAP, which was calculated using parameters from Ref. [22] and a well-known formula of Mott and Seitz [23]. Yet the scintillation light yields of LuAP:Ce and YAP:Ce, presented in Fig. 4b and c, show large variations in exactly the same range of temperatures where photoluminescence efficiency stays nearly constant.

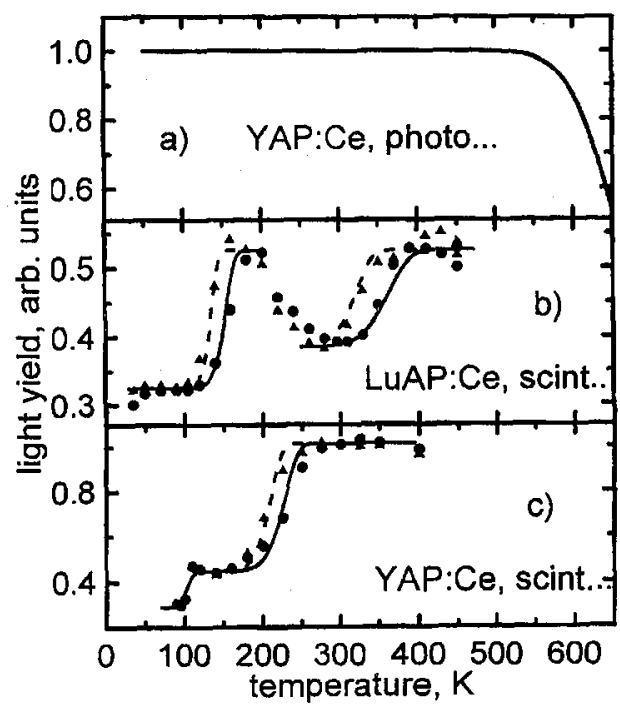

Fig. 4. Plots of photoluminescence and scintillation light yields against temperature. The photoluminescence efficiency curve for YAP:Ce (a), was calculated using parameters for YAP:Ce from the literature with no account of phonon contribution to the radiative lifetime (see text). Experimental scintillation $L Y$ points in (b) and (c) are shown by circles ( $0.5 \mu$ s shaping time) and by triangles ( $4 \mu$ s shaping time for LuAP:Ce and $2 \mu \mathrm{s}$ for YAP:Ce). Solid and dashed lines represent fits to experimental points calculated from the model (see text).

Although measured scintillation light yield curves for LuAP:Ce and YAP:Ce appear very different they help explain why the RT yield of the former is so much lower. At this particular temperature YAP:Ce has already attained its maximum value while LuAP:Ce is barely at $73 \%$ of its maximum value, which it does not reach until at least $380 \mathrm{~K}$. At this temperature the yield of LuAP:Ce, corrected 
to a value of $12,900 \mathrm{ph} / \mathrm{MeV}$ for the loss to the deep traps (measured at RT by Bartram et al. [18]), would increase to $17,700 \mathrm{ph} / \mathrm{MeV}$, almost exactly the same as that of YAP:Ce at RT. When measured at temperatures where both materials have reached their maximal values, the disparity between the scintillation light yields of the two closely related aluminates disappears. Both curves, despite their differences at lower temperatures, converge at higher temperatures.

As we have demonstrated in two recent papers $[15,16]$, the variations in scintillation $L Y$ of LuAP:Ce and YAP:Ce below $400 \mathrm{~K}$ can be consistently explained by a model incorporating a $\mathrm{Ce}^{3+}$ recombination center and a number of electron traps. The first key is to recognize that in scintillation, unlike steady state photoluminescence, the concentration of emitting species does not remain constant. The scintillation $L Y$ points, presented in Fig. $4 \mathrm{~b}$ and c, give an average amount of scintillation light produced in a single event (one gamma particle stopped) and integrated over a finite time after the beginning of the scintillation pulse. This integration time is equal to $2.35 \tau_{\mathrm{sh}}$, where $\tau_{\mathrm{sh}}$ is the shaping time, typically 0.5 to $4 \mu \mathrm{s}$. Since the contribution from a trap strongly depends on temperature via its cross-section for charge carrier emission, the total light yield must change accordingly. Each of the steps in the plots shown in Fig. 4b and c, where $L Y$ jumps to a higher value as temperature increases, corresponds to its own particular trap characterized by a unique pair of values for the energy depth $E$ and frequency factor $s$.

Let us consider this in more detail. The trap emission rate $p$, or filled trap lifetime $\tau$, is given by the well-known formula

$$
p=\frac{1}{\tau}=s \exp \left(-\frac{E}{k_{\mathrm{B}} T}\right),
$$

where $s$ is the frequency factor, $E$ the trap depth, and $k_{\mathrm{B}}$ the Boltzmann constant. This tells us that at low enough temperatures the contribution to the scintillation pulse coming from a trap described by a depth $E$ and frequency factor $s$ will be characterized by a long decay time (equal to $\tau$ ) and a relatively small zero-time amplitude, $A$. At high enough temperatures the situation will be reversed, with a short decay time and a large zero-time amplitude. This is because, to a first approximation, temperature does not affect the total contribution from a given trap, only the time in which it is made. In the absence of any other losses, the fraction of the total light yield delivered by the trap will be determined solely by the distribution of electrons between the traps and the $\mathrm{Ce}^{4+}$ ions previously formed by hole capture, established shortly after a $\gamma$-particle was stopped in the material $[15,16]$. It is now clear that at low temperatures the contribution from the trap to the measured scintillation $L Y$ must be practically zero, but above some threshold temperature will begin to rise rapidly, eventually reaching its maximal value. While a simple formula (using the zero-time amplitude approximation) [14] is generally adequate to describe this behavior, we show in Refs. [15] and [16] that the correct formula describing the $L Y$ vs. $T$ dependence assumes the form

$$
L Y=L Y_{0}\left\{a+b \frac{p \tau_{\mathrm{rad}}}{p \tau_{\mathrm{rad}}-1}\left[1+\frac{1}{p \tau_{\mathrm{rad}}}\left(\exp \left(-2.35 \tau_{\mathrm{sh}} p\right)-1\right)\right]\right\},
$$

where $L Y_{0}$ is the sum of electrons generated by a $\gamma$-particle that went initially to either $\mathrm{Ce}^{4+}$ ions or traps (which is also the projected "trap-free" material light 
yield), while the branching coefficients $a$ and $b$ describe the initial distribution of electrons between the $\mathrm{Ce}^{4+}$ ions (creating excited $\mathrm{Ce}^{3+}$ ions) and the relevant trap. The parameter $p$, as before, stands for the thermally activated trap emission rate, $\tau_{\mathrm{sh}}$ is the shaping time, and $\tau_{\mathrm{rad}}$ is the $\mathrm{Ce}^{3+}$ radiative lifetime.

Solid and dashed lines in Fig. $4 \mathrm{~b}$ and $\mathrm{c}$ represent fits to experimental points calculated using formula (3) for $\tau_{\text {sh }}$ equal to 0.5 and $4 \mu$ s (LuAP:Ce), and 0.5 and $2 \mu$ s (YAP:Ce). The decrease in the $L Y$ at temperatures between 200 and $280 \mathrm{~K}$ in LuAP:Ce is most likely due to a thermally activated carrier capture by the deeper of the two shallow LuAP:Ce traps, essentially preventing it from filling unless the temperature is high enough. This part of the curve has not been fitted to the model as this would require introduction of another parameter, independent and unrelated, introducing undesirable complications. Since all the other traps are shallower, their respective energy barriers are expected to be too low to interfere with charge capture [15]. The parameters obtained from the $L Y$ vs. $T$ curves for traps in LuAP:Ce and YAP:Ce are summarized in Table III. We have also included in this table the room temperature trap lifetimes, calculated from formula (2). Note that moderate variances in trap depths translate into vastly different trap lifetimes; this must be of major importance for scintillation kinetics, to be discussed shortly.

TABLE III

Summary of shallow trap parameters for YAP:Ce and LuAP:Ce, obtained from the scintillation $L Y$ vs. $T$ measurements. The direct and trap-mediated contributions to the light yield (represented by parameters $a$ and $b$ ) are normalized by setting the high-temperature $L Y$ to unity. The values of frequency factors were assumed constant at $4.8 \times 10^{12} \mathrm{~s}^{-1}$. The values of $E$ were obtained from the fits. The trap lifetime $\tau$ at room temperature $(295 \mathrm{~K})$ was calculated from the trap parameters $s$ and $E$.

\begin{tabular}{c|c|c|c|c|r}
\hline \hline Trap & Direct $(a)$ & Via trap $(b)$ & $E[\mathrm{eV}]$ & $\ln s$ & $\tau[\mathrm{ns}]$ \\
\hline YAP, trap1 & 0.27 & 0.16 & 0.140 & 29.2 & 0.051 \\
YAP, trap 2 & & 0.57 & 0.309 & 29.2 & 39 \\
LuAP, trap1 & 0.45 & 0.28 & 0.210 & 29.2 & 0.8 \\
LuAP, trap 2 & & 0.27 & 0.495 & 29.2 & 58,000
\end{tabular}

We should emphasize that the difference in appearance of $L Y$ vs. $T$ curves of LuAP:Ce and YAP:Ce is only superficial, deriving entirely from the fact that traps in LuAP:Ce are deeper than their counterparts in YAP:Ce. This is the only reason why the entire curve in LuAP:Ce is shifted toward higher temperatures, accidentally putting a local minimum at room temperature. The only major difference between the two curves, the downward trend between 200 and $280 \mathrm{~K}$ in LuAP:Ce, is similarly attributed to the greater depth of the relevant trap, allowing a higher energy barrier for trapping and a concomitant increase in charge carrier capture rate as the temperature rises. Since YAP:Ce traps are shallower, such an effect is not observed in that material. 


\section{Low temperature thermoluminescence}

To verify the existence of traps responsible for variations in the $L Y$ vs. $T$ curves in the aluminates, we recall the low temperature thermoluminescence (ltTL) glow curves that have been measured before [24, 16]. In Fig. 5 the measured glow curves represented by small circles are compared with the four simulated glow curves shown by solid lines. The simulated curves were calculated from the first order Randall and Wilkins formula with trap parameters derived from the $L Y$ vs. $T$ fits for LuAP:Ce and YAP:Ce. For each aluminate one of the simulated peaks falls reasonably close to the measured glow peak. In LuAP:Ce there is also a second much weaker experimental glow peak which has no counterpart in YAP:Ce. Both the weakness of the second experimental peak in LuAP:Ce and its absence in YAP:Ce may be due to the inability of very shallow traps to retain a significant population of electrons and to make a measurable contribution to the ltTL. A slight adjustment of parameters leading to good and consistent match between measurements and theory in both experiments, ltTL and $L Y$ vs. $T$, is possible, as demonstrated in Refs. [15] and [16].

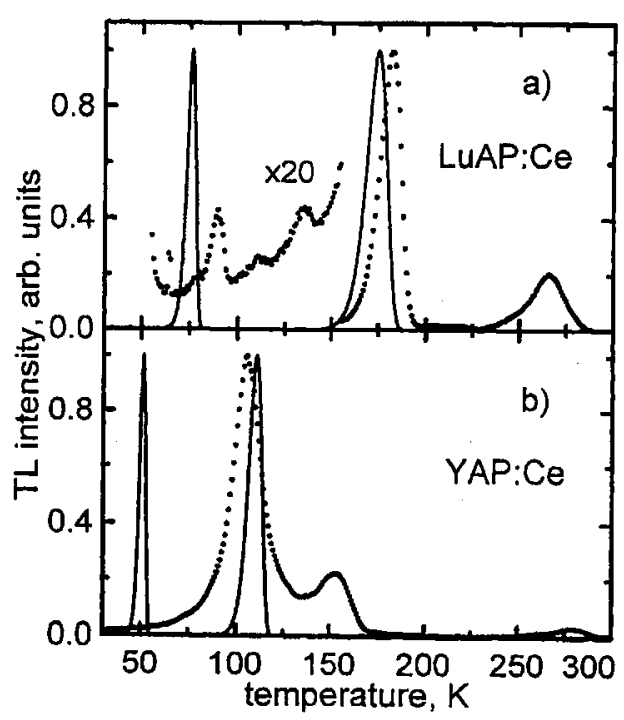

Fig. 5. Measured and simulated glow curves of LuAP:Ce (a) and YAP:Ce (b) at temperatures below RT. The heating rate was $0.15 \mathrm{~K} \mathrm{~s}^{-1}$. Experimental points are shown by circles while solid lines represent simulated glow curves calculated from a simple first-order Randall and Wilkins formula using parameters obtained from the $L Y$ vs. $T$ fits.

In both materials the ltTL glow curves reveal the existence of the third minor shallow trap. This trap, however, is too close in depth to the major trap to be separately resolved in the $L Y$ vs. $T$ experiment. All three traps follow the pattern previously noted, where the traps in LuAP:Ce are deeper than the corresponding traps in YAP:Ce. We note also that the widths of the experimental glow curves 
of YAP:Ce suggests that each trap would be better described by a distribution of energies rather than a single discrete value [16]. The effect of the third trap on scintillation performance of YAP:Ce has been discussed before [16].

\section{Scintillation kinetics}

Since all of the traps that glow at temperatures below $300 \mathrm{~K}$ must be able to capture and release electrons at that particular temperature, it is reasonable to assume that their effects may be seen at room temperature as well. This would take the form of diverting part of the energy deposited in the crystal by a high energy particle into slower components, thereby modifying the scintillation time profile. This is clearly the case of the deeper traps in LuAP:Ce and YAP:Ce, characterized by lifetimes of $58 \mu \mathrm{s}$ and $39 \mathrm{~ns}$, respectively (see Table III). Closer examination of even the simplest scintillator model, however, reveals that very shallow, short lived traps can alter the rising part of the scintillation time profile as well [15].

In a system consisting of one recombination center and one trap and evolving under the first-order kinetics the scintillation time profile is given by [15]

$$
\begin{aligned}
I(t) & =I_{0}\left\{\frac{a}{\tau_{\text {rad }}-\tau_{0}}\left[\exp \left(-\frac{t}{\tau_{\text {rad }}}\right)-\exp \left(-\frac{t}{\tau_{0}}\right)\right]\right. \\
& \left.+\frac{b}{\tau-\tau_{\text {rad }}}\left[\exp \left(-\frac{t}{\tau}\right)-\exp \left(-\frac{t}{\tau_{\text {rad }}}\right)\right]\right\} .
\end{aligned}
$$

In this formula the branching coefficients $a$ and $b$ describe the normalized contributions due to direct and trap-mediated recombination channels, $\tau_{\text {rad }}$ and $\tau$ are the $\mathrm{Ce}^{3+}$ radiative time and the trap lifetime, respectively, and $\tau_{0}$ has been introduced to describe the rising part of the direct term.

In a lightly doped material the direct term cannot truly be instantaneous, but rather must reflect the rate of the fastest possible channel of radiative recombination via the $\mathrm{Ce}^{3+}$ ion. This involves direct recombination of electrons in the conduction band with those $\mathrm{Ce}^{4+}$ ions that captured holes on a time scale too short to measure, shortly after the high energy particle was stopped in the material. The time constant $\tau_{0}$ will, therefore, describe the slowest of those fast early stages of kinetics, involving the decay of the initial highly nonequilibrium electron and hole populations in the concluction and valence bands, the polaron effects (relaxation), carrier (polaron) diffusion (hopping) and/or the decay of the Ce-bound exciton, which is the last step preceding the emission [15].

The possibility that shallow traps might modify the rising part of the scintillation time profile follows directly from the formula (4). Notice that for trap lifetimes $\tau$ shorter than $\tau_{\text {rad }}$ the two exponents in the second term of the right-hand expression (4) change signs and, consequently, the decay is described by the radiative lifetime $\tau_{\text {rad }}$ and the rise by $\tau$, the trap lifetime. It is, therefore, tempting to examine the possibility that some of the shallow traps in LuAP:Ce and YAP:Ce, identified with the help of the $L Y$ vs. $T$ experiment, might be responsible for the apparent differences in rise times in scintillation profiles of the two materials.

In Fig. 6 we depict by a sequence of points two-exponential simulations of experimental scintillation time profiles for LuAP:Ce and YAP:Ce

$$
I(t) \propto\left[\exp \left(-\frac{t-t_{0}}{\tau_{\text {eff }}}\right)-\exp \left(-\frac{t-t_{0}}{\tau_{0}}\right)\right]
$$




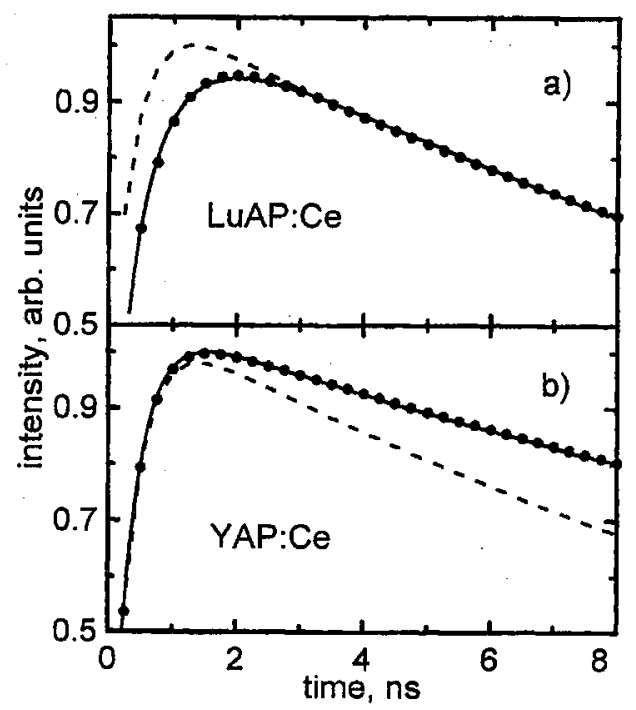

Fig. 6. Scintillation time profiles of LuAP:Ce (a) and YAP:Ce (b). Points represent a two-exponential simulation of experimental profiles characteristic of the "real" materials. Solid lines show simulated time profiles calculated from the one-trap model discussed in the text; dashed lines show simulated profiles of the "trap-free" materials.

where, after Derenzo [13] and Moszynski et al. [25], we assign $\tau_{0}$ values of $0.600 \mathrm{~ns}$ for LuAP:Ce and $0.350 \mathrm{~ns}$ for YAP:Ce, and values of the effective scintillation decay time $\tau_{\text {eff }}$ of $16.8 \mathrm{~ns}$ (the radiative decay time) for LuAP:Ce and $28 \mathrm{~ns}$ for YAP:Ce, reasonably within reported limits [26]. The parameter $t_{0}$ compensates for the somewhat arbitrary choice of zero time in the fitting procedure.

In this approximation of the real experimental scintillation profile we neglect longer components from both shallow and deeper traps since we are interested only in the effect of the short-lived traps. We also assume that the $0.350 \mathrm{~ns}$ rise time of YAP:Ce is not determined by any of traps listed in Table III, but rather is the intrinsic rise time characteristic of the direct component in both YAP:Ce and LuAP:Ce. Under these assumptions only one trap in each material, that is the $0.8 \mathrm{~ns}$ trap in LuAP:Ce and the $39 \mathrm{~ns}$ trap in YAP:Ce are relevant, the others being too short- or too long-lived to have any influence on scintillation profiles in the time range of interest.

The solid lines in Fig. 6 present the fits to the sequence of points using the one-trap formula (4) with the values of branching parameters $a$ and $b$ obtained from the $L Y$ vs. $T$ fits: 0.45 and 0.28 for LuAP:Ce, and 0.49 and 0.51 for YAP:Ce. Under this choice the contribution of the shallower $51 \mathrm{ps}$ lifetime trap of YAP:Ce is included in the direct component while the contribution of the deeper $58 \mu$ s trap of $\mathrm{LuAP}: \mathrm{Ce}$ is ignored. To find the best fit we varied the scaling factor $I_{0}$, zero time $t_{0}$ and the trap lifetime $\tau$. The trap lifetime found from the fit is $0.9 \mathrm{~ns}$ for LuAP:Ce and $62 \mathrm{~ns}$ for YAP:Ce. These values ought to be compared to the values obtained from the $L Y$ vs. $T$ fits and given in Table III. Clearly, the agreement is quite reasonable. 


\section{Conclusions}

The studies of radioluminescence from two isostructural scintillator materials, LuAP:Ce and YAP:Ce, consistently point to the recombination of charge carriers via the $\mathrm{Ce}^{3+}$ ions as the dominant mechanism of radioluminescence in these materials. The results of experimental studies, including measurements of high temperature thermoluminescence, scintillation light yield as function of temperature, low temperature thermoluminescence and scintillation time profiles, are interpreted in the frame of a simple kinetic model that includes one recombination center and one trap. In particular we have established that the majority of electrons that recombine with $\mathrm{Ce}^{4+}$ ions have already spent some time in electron traps, which we have identified and characterized during the course of this study. While the deep traps are responsible for the scintillation light loss, shallow traps are also responsible for some unexpected and poorly understood features of the two materials, including disparate scintillation light yields and scintillation time profiles at room temperature. All the traps found, except for a single dominant one unique to LuAP:Ce, are present in both materials, with those in YAP:Ce consistently shallower than their counterparts in LuAP:Ce. Although the exact nature of these traps remains unknown it is clear that their elimination would significantly improve the performance of both materials.

\section{Acknowledgments}

The work presented in this paper was performed in the frame of collaboration funded by grants from the US Department of Energy (DE-FG-02-90ER61033), the European Community (ERBCIPDCT-940037), and the Committee for Scientific Research (Poland) (2P03B04914). The experiments were conducted at the Chemistry Department, Boston University and the Institute of Physics, N. Copernicus University, by K.R. Przegietka and W. Drozdowski (measurements and fits to htTL and ITD experiments), D. Wisniewski (early ltTL experiments, design and construction of $L Y$ vs. $T$ setup and early experiments), and J. Glodo ( $L Y$ vs. $T$, ltTL experiments), to all of whom I offer my special thanks. I am grateful to M.H. Randles of Litton Airtron and M. Kokta of Union Carbide Corp., who grew the crystals of LuAP:Ce and YAP:Ce, respectively, and to T. Piters of Universidad de Sonora, Hermosillo, Mexico, who wrote the TL-fitting procedure TAMTAM. I am also very grateful to Profs. A. Lempicki and C. Brecher of Boston University, and Prof. H.L. Oczkowski of N. Copernicus University, for their continuous support and many fruitful discussions.

\section{References}

[1] G.F.J. Garlick, in: Luminescence of Inorganic Solids, Ed. P. Goldberg, Academic Press, New York 1966, p. 685.

[2] C.L. Melcher, J.S. Schweitzer, IEEE Trans. Nucl. Sci. 39, 502 (1992).

[3] A. Lempicki, M.H. Randles, D. Wisniewski, M. Balcerzyk, C. Brecher, A.J. Wojtowicz, in: 1994 IEEE Nucl. Sci. Symp. 8 Med. Imag. Conf. Record, Ed. R.C. Trendler, IEEE Inc., Piscataway, NJ 1995, p. 307; A. Lempicki, M.H. Randles, D. Wisniewski, M. Balcerzyk, C. Brecher, A.J. Wojtowicz, IEEE Trans. Nucl. Sci. 42, 280 (1995). 
[4] W.P. Trower, in: Mater. Res. Soc. Symp. Proc., Vol. 348, Eds. M.J. Weber, P. Lecoq, R.C. Ruchti, C. Woody, W.M. Yen, R. Zhu, Materials Research Society, Pittsburgh 1994, p. 131.

[5] A. Lempicki, A.J. Wojtowicz, C. Brecher, in: Wide-Gap Luminescent Materials: Theory and Applications, Ed. S.R. Rotman, Kluwer Academic Publ., Boston 1997, p. 235.

[6] D.J. Robbins, P.J. Dean, Adv. Phys. 27, 499 (1978).

[7] C.C. Klick, J.H. Schulman, Solid State Phys. 5, 97 (1957).

[8] R.G. Kaufman, W.B. Hadley, H.N. Hersh, IEEE Trans. Nucl. Sci. 17, 82 (1970).

[9] A.J. Wojtowicz, A. Lempicki, D. Wisniewski, M. Balcerzyk, C. Brecher, IEEE Trans. Nucl. Sci. 43, 2168 (1996).

[10] K.S. Song, R.T. Williams, Self-trapped Excitons, Springer-Verlag, Berlin 1993, p. 42 .

[11] M.V. Korzhik, W.P. Trower, Appl. Phys. Lett. 66, 2327 (1995).

[12] C. Pedrini, D. Bouttet, C. Dujardin, A. Belsky, A. Vasil'ev, in: Proc. Int'l Conf. on Inorganic Scintillators and Their Applications, Eds. P. Dorenbos, C.W.E. van Eijk, Delft University Press, Delft 1996, p. 103.

[13] S.E. Derenzo, private communication, to be published.

[14] A.J. Wojtowicz; W. Drozdowski, D. Wisniewski, K. Wisniewski, K.R. Przegietka, H.L. Oczkowski, T.M. Piters, Radiation Measurements 29, 323 (1998).

[15] A.J. Wojtowicz, J. Glodo, W. Drozdowski, K.R. Przegietka, J. Lumin. 79, 275 (1998).

[16] A.J. Wojtowicz, J. Glodo, A. Lempicki, C. Brecher, J. Phys., Condens, Matter 10, 8401 (1998).

[17] M. Moszynski, M. Kapusta, M. Mayhugh, D. Wolski, S.O. Flyckt, IEEE Trans. Nucl. Sci. 44, 1052 (1997).

[18] R.H. Bartram, D.S. Hamilton, L.A. Kappers, A. Lempicki, J. Lumin. 75, 183 (1997).

[19] S.W.S. McKeever, Thermoluminescence of Solids, Cambridge University Press, Cambridge 1985, p. 98.

[20] A.J.J. Bos, T.M. Piters, J.M.G. Ros, A. Delgado, Rodiat. Protect. Dosimetry 47, 473 (1993).

[21] J.T. Randall, M.H.F. Wilkins, Proc. R. Soc. Lond. A 184, 366 (1945).

[22] L.L. Lyu, D.S. Hamilton, J. Lumin. 48\&49, 251 (1991).

[23] B. Di Bartolo, Optical Interactions in Solids, Wiley, New York 1968.

[24] A. Lempicki, J. Glodo, Nucl. Instrum. Methods Phys. Res. A 416, 333 (1998).

[25] M. Moszynski, M. Kapusta, D. Wolski, W. Klamra, B. Cederwall, Nucl. Instrum. Methods Phys. Res. A 404, 157 (1998).

[26] M.V. Korzhik, O.V. Misevich, A.A. Fyodorov, Nucl. Instrum. Methods. Phys. Res. B 72, 499 (1992). 